\title{
Unspecified Method of Administration
}

National Cancer Institute

\section{Source}

National Cancer Institute. Unspecified Method of Administration. NCI Thesaurus. Code C149701.

The technique of administration of a pharmaceutical product by an unspecified method. 